\title{
REgULACIÓN DE LA MOVILIDAD URBANA EN EL ÁREA METROPOLITANA DE MENDOZA
}

\author{
Lía Martínez* \\ María Emilia García Schilardi* \\ María Laura Devito ${ }^{* * *}$ \\ MARISA DÍAz ${ }^{* * * *}$
}

\section{Resumen}

El paradigma de la movilidad urbana sostenible requiere, para ser posible, capacidades institucionales, políticas adecuadas, y un marco regulatorio que las contenga. El presente trabajo tiene como objetivo aportar al conocimiento de la regulación de la mo- vilidad urbana en el área metropolitana de Mendoza. Para ello, se evalúa la normativa vigente en movilidad a través de indicadores que se enmarcan en tres aspectos clave: organización político-institucional, sistema urbano y esquema de financiamiento. Se pretende dar cuenta de la existencia o no de capacidades de regulación contenidas en el paradigma de

* Licenciada en Ciencia Política y Administración Pública. Alumna de la Maestría en Planificación y Gestión de Transporte, Facultad de Ingeniería, UBA, Buenos Aires (Argentina). [lia.e.martinez@gmail.com]

** Magíster en Economía Urbana y en Ordenamiento Territorial. Candidata al Doctorado en Ordenamiento del Territorio y Desarrollo Sostenible, Universidad Nacional de Cuyo. Miembro del equipo de trabajo del Programa de Movilidad Sostenible. Instituto de Ciencias Ambientales, Universidad Nacional De Cuyo, Mendoza (Argentina). [maria-emilia-garcia@hotmail.com]

*** Arquitecta. Estudiante de la maestría en Ordenamiento Territorial con Orientación en Planificación, Universidad Nacional de Cuyo, Argentina. Docente Facultad de Ingeniería, Universidad Nacional de Cuyo, Mendoza (Argentina). [malauradevito@hotmail.com]

****Estudiante de la licenciatura en Ciencia Política y Administración Pública, Facultad de Ciencias Políticas y Sociales, Universidad Nacional de Cuyo, Mendoza (Argentina). [madi1903@gmail.com]

Recibido: 1 de julio de 2016 / Modificado: 29 de agosto de 2016 / Aceptado: 1 de septiembre de 2016

Para citar este artículo

Martínez, L., García Schilardi, M. E., Devito, M. L. y Díaz, M. (2016). Regulación de la movilidad urbana en el área metropolitana de Mendoza. opera, 19, pp. 161-181.

DOI: http://dx.doi.org/10.18601/16578651.n19.09 
movilidad sostenible. Entre los resultados se detecta la ausencia de una política de movilidad urbana sostenible, la inexistencia de integralidad sectorial y coordinación entre la planificación del sistema urbano y del servicio de transporte colectivo, y un esquema de financiamiento tendiente a facilitar los modos sostenibles de transporte pero no pensado con ese objetivo.

Palabras clave: movilidad urbana sostenible, organización institucional, sistema urbano, financiamiento, transporte.

\section{URBAN MOBILITY REGULATION IN METROPOLITAN AREA OF MENDOZA}

\section{Abstract}

Achieving the paradigm of sustainable urban mobility requires institutional capacities, appropriate policies and a regulatory framework that contains them. This work aims to contribute to the knowledge of the regulation of urban mobility in the metropolitan area of Mendoza. To this end, the current mobility regulations are assessed through indicators that are classified into three key areas: institutional and political organization, urban system and financial setup. The purpose is to account for the existence, or not, of regulatory capacities contained in the paradigm of sustainable mobility. Among the results, the absence of a policy of sustainable urban mobility is noteworthy, as well as the lack of sectorial coordination. Also of note is the absence of coordination between the urban planning system and the public transport pro- vision. Lastly, in the financial sector, the results point to a promotion of sustainable transport modes but without such an explicit purpose.

Key words: Sustainable urban mobility, institutional organization, urban system, transport financing.

\section{INTRODUCCIÓN}

El paradigma de la movilidad urbana sustentable se basa en la promoción de medios de transporte colectivos y no motorizados, coordinados y articulados entre sí, como elementos claves para el desarrollo de ciudades sustentables. Este paradigma asume un modelo de movilidad enfocado en las personas que se desplazan poniendo los medios de transporte a disposición de las mismas. Esto supone un cambio en la concepción de la movilidad en tanto que ya no se enfoca solamente en la eficiencia de los modos de transporte, sino que también tiene en cuenta las necesidades de desplazamiento de la población y su relación con el ambiente y la estructura social, espacial, económica e institucional del territorio. Esta estrategia requiere, para ser posible, capacidades institucionales y políticas adecuadas y un marco regulatorio que la contenga.

El presente trabajo tiene como objetivo aportar al conocimiento de la regulación de la movilidad urbana en el área metropolitana de Mendoza (AMM). Para ello se evalúa la normativa vigente en movilidad por medio del análisis de indicadores que se enmarcan en tres aspectos clave: organización políticoinstitucional, sistema urbano y esquema de financiamiento. Se pretende dar cuenta de la 
existencia o no de estrategias contenidas en el paradigma de movilidad sostenible que permitan el desarrollo coordinado y eficiente de las dimensiones social, económica y ambiental de las ciudades.

Se utilizó una metodología basada en la revisión documental nacional e internacional. A partir de ella se elaboraron indicadores que tomaron la normativa local como base de información para desarrollarlos. A su vez, los antecedentes internacionales se emplearon para definir los parámetros deseados de dichos indicadores conforme el paradigma de movilidad urbana sostenible.

Entre los resultados se detecta la ausencia de una política de movilidad urbana sostenible, la inexistencia de integralidad sectorial y coordinación entre la planificación del sistema urbano y el servicio de transporte colectivo, $\mathrm{y}$ un esquema de financiamiento tendiente a facilitar los modos sostenibles de transporte pero no pensado con ese objetivo.

\section{MARCO TEÓRICO}

La movilidad se materializa a través del transporte y este se configura en diversos modos. Actualmente, en muchas ciudades del mundo la movilidad se desarrolla con base en la utilización del automóvil particular. Las infraestructuras, las nuevas tecnologías y las costumbres urbanas se orientan casi exclusivamente al uso del vehículo individual. Este modelo de movilidad no resulta óptimo ya

1 Véase http://app.idu.gov.co/seccion_metro_ASP/ que tan altos índices de motorización generan congestión, contaminación, aumento de los accidentes de tránsito y un uso no eficiente del espacio urbano. Asimismo, dicho modelo ha fomentado la expansión irracional y espontánea de las ciudades con una consecuente fragmentación espacial y segregación social.

Como respuesta a esta situación, surge el paradigma de movilidad urbana sostenible que asume un modelo de movilidad enfocado en las personas que se desplazan, y no solo en los modos de transporte. Este modelo supone un cambio en la concepción de la movilidad en tanto que ya no se enfoca solamente en la eficiencia de los modos de transporte sino también en las necesidades de desplazamiento de la población y su relación con el ambiente y la estructura social, espacial, económica e institucional del territorio (Acevedo y Bocarejo, 2009).

El Libro Verde del Transporte Urbano de la Comisión Europea (2007) refleja este cambio de paradigma al promover un enfoque integrado que posibilite el desarrollo económico de las ciudades, favorezca una mejor calidad de vida de sus habitantes y promueva la conservación y protección del medio ambiente.

La movilidad es un derecho sujeto a ciertos límites referidos a los impactos asociados a cada medio y sistema de transporte. La movilidad sostenible ${ }^{11}$ es aquella capaz de satisfacer las necesidades de la sociedad de moverse libremente, acceder, comunicarse, comercializar o establecer relaciones sin sa-

1 Según el World Business Council for Sustainable Development (WBCSD). 
crificar otros valores humanos o ecológicos básicos actuales o del futuro.

Este modelo se constituye a través de una estrategia propuesta por el Banco Interamericano de Desarrollo (Vicentini, 2013):

- Evitar viajes: integrar los usos del suelo con la planificación del transporte, promover los usos mixtos, la consolidación urbana y un nivel aceptable de densidad de población, gestionar la demanda de viajes.

- Promover modos más sostenibles: racionalizar el uso del automóvil particular, pasar así a modos colectivos eficientes, y promover los modos de transporte no motorizado.

- Mejorar la eficiencia de los viajes: planificar una red multimodal, utilizar nuevas tecnologías de vehículos y combustibles, mejorar la gestión de la red vial, aplicar nuevas prácticas logísticas.

Esta estrategia permite el desarrollo de las dimensiones social, económica y ambiental de las ciudades. Una red de transporte multimodal coordinado, eficiente y con tecnologías limpias facilita el acceso de toda la población a sus lugares de trabajo y estudio, a los lugares donde se prestan servicios de salud y demás servicios urbanos (UiTp, 2004; Zaragoza et al., 2009). Favorece la consolidación de la ciudad, la integración social, el desarrollo de actividades económicas y el control de emisiones contaminantes. El paradigma de movilidad urbana sostenible hace foco en las personas y sus necesidades, y pone los medios de transporte a disposición de las mismas. Esta estrategia requiere, para ser posible, capacidades institucionales y políticas adecuadas, y un marco regulatorio que las contenga. Una política de movilidad integral y sostenible plantea a las autoridades políticas la necesidad de desarrollar mayor capacidad de coordinación y de cooperación entre sectores y entre niveles de Gobierno del orden nacional y local. Además, tiene como requisito la incorporación de la participación del sector privado y la sociedad civil (universidades, organizaciones de usuarios, ambientalistas, etc.) para el desarrollo de visiones de largo plazo.

\section{METODOLOGÍA}

Como primer paso para el desarrollo de esta investigación se analizaron antecedentes internacionales y nacionales de implementación de modelos de movilidad urbana sostenible que sirvieron como ejemplo para las propuestas por realizarse en el caso de estudio. En particular, se indagó sobre tres aspectos clave de estos antecedentes, detallando sus características positivas y negativas:

1. Esquema de organización política e institucional.

2. Relación del sistema de movilidad con el sistema urbano.

3. Esquema de financiamiento y tarificación.

En función de ello, la evaluación de la regulación de la movilidad del AMM, en clave de las estrategias de movilidad urbana sostenible, se realizó considerando estos tres aspec- 
tos. Para cada uno de ellos se seleccionaron indicadores representativos que se enuncian a continuación:

1. Esquema de organización política e institucional: articulación intergubernamental para la planificación de la movilidad urbana provincia-municipio, regulación del uso de los diferentes modos de transporte de personas, regulación de los usos del espacio vial por modos motorizados y no motorizados, y regulación del tipo de información disponible para los usuarios de los servicios de: transporte público masivo, movilidad no motorizada y estacionamiento medido en el AMM.

2. Relación del sistema de movilidad con el sistema urbano: tributación diferenciada al suelo urbano vacante (suv), intensidad de ocupación (FOs у FOт), zonificación de usos de suelo y distribución de usos de suelo.

3. Esquema de financiamiento y tarificación: integración tarifaria entre modos, trasbordos sin costo, recaudación / distribución por único operador, proporción de financiamiento mediante subsidios (proporción de financiamiento a través de tarifa), y organismo que fija la tarifa.

Cada indicador se analizó siguiendo la metodología propuesta por un informe de la Agencia de Ecología Urbana de Barcelona (2010). Dicha propuesta sugiere estudiar cada indicador según las siguientes características: nombre, objetivo, definición, metodología, categorías, parámetros de evaluación y discusión de resultados. A los fines de este artículo no se expondrá el desarrollo de cada indicador, sino el resultado más importante contenido en la discusión de resultados.

Es oportuno mencionar que el АMм se ubica en la provincia de Mendoza, Argentina. La misma se localiza en el centro norte de la provincia y su territorio se encuentra dividido administrativamente en seis departamentos: Capital, Godoy Cruz, Las Heras, Luján de Cuyo, Maipú y Guaymallén (figura 1). El sistema de transporte colectivo que opera en esta área se compone de tres modos: ómnibus, trolebús y tranvía. El servicio de ómnibus es operado por empresas privadas, adjudicado a través de licitaciones; en cambio, los servicios de trolebús y tranvía son prestados por una empresa estatal. Complementariamente, existen servicios de taxis, remises y contratados, $\mathrm{y}$ hay presencia, obviamente, de servicios particulares como la motocicleta y el automóvil.

\section{RESULTADOS}

Para cada indicador se detectaron los siguientes resultados:

\section{Indicadores de la dimensión política institucional}

\section{Articulación intergubernamental para la planificación de la movilidad urbana provincia-municipio}

Del total de los instrumentos que permitirían institucionalizar la articulación intergubernamental para la planificación de la movilidad urbana se observa que el AMM 
FIGURA 1. ÁREA METROPOLITANA DE MENDOZA

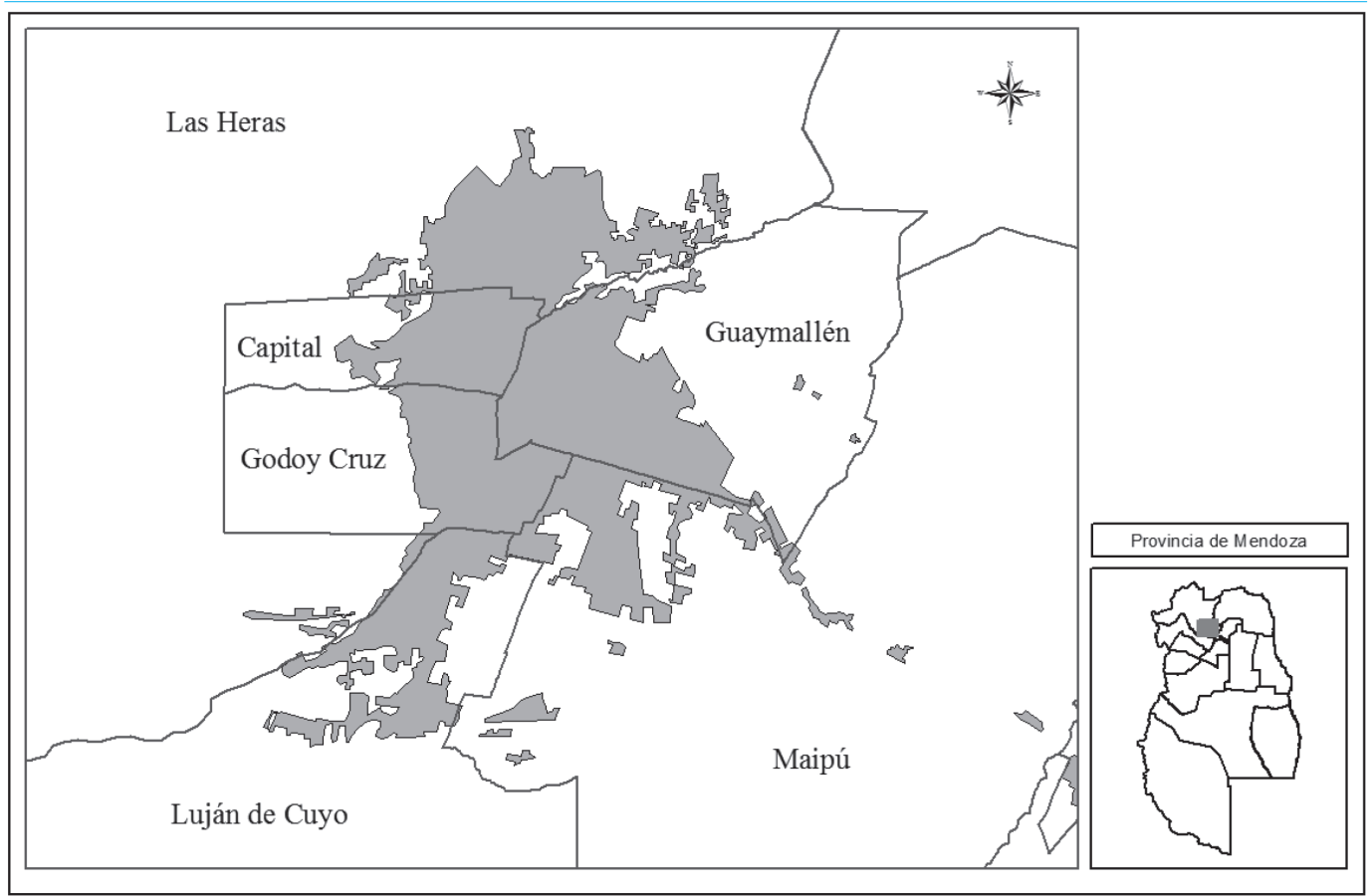

Ley enda

$\square$ Departamentos

Área Metropolitana de Mendoza, Argentina

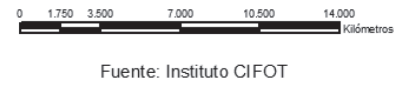

Fuente: Instituto CIFOT, UNCuyo (2015).

cuenta únicamente con un órgano consultivo, con funciones establecidas hace 24 años. No tiene convenios, actas-acuerdo o leyes que le permitan a la autoridad coordinadora del transporte, contar con capacidad para llevar adelante dicha articulación.

Además, se observa un alto nivel de complejidad para la articulación interjurisdiccional, propia de los Estados federales. Con la Ley Provincial 6082/1993 de Tránsito y Transporte, culmina un proceso de acuerdos para que la competencia sobre la materia pase de ser municipal a provincial.

Por su parte, la Ley Marco de Transporte Público de Pasajeros 7412/2005 es la principal norma reguladora del transporte terrestre en Mendoza y expresa que:

La administración, planificación y regulación del transporte público de pasajeros, en todas sus formas y modalidades, y el control de la operación del sistema en el ámbito del territorio de la Provincia y de las compe- 
tencias que le son propias al Estado provincial, como servicio público esencial para el desarrollo humano y económico.

Se debe interpretar que la norma regula la prestación de servicios públicos regulares de transporte. Quedan sin ser abarcados por la normativa vigente servicios de préstamo público de bicicletas como los implementados por la Secretaría de Servicios Públicos.

\section{Regulación del uso de los diferentes modos de transporte de personas}

Del conjunto de modos de transporte que conviven en el AMM, observamos que solo aquellos motorizados y colectivos (ómnibus, trolebús y tranvía) tienen una regulación aceptable en el marco de su aporte a la sostenibilidad. Sin embargo, el alcance de la regulación no es pertinente ya que no explicita el rol del usuario como sujeto de derecho, al cual el Estado debe garantizarle la movilidad.

En un tratamiento menor por la normativa provincial y municipal, nos encontramos con los servicios de taxis, remises y contratados con un fin específico; motos y auto particular. Ninguna norma hace expresos los objetivos de movilidad urbana sostenible, como por ejemplo, desincentivar el uso del auto particular o la moto. El único caso es la Ordenanza 3877/2014, de la Municipalidad de la Ciudad de Mendoza (aún no reglamentada ni implementada), que restringe el acceso en ciertos días y horarios de vehículos particulares a su territorio. La Municipalidad de Godoy Cruz, cuenta con normativa sobre el uso de la bicicleta, pero mantiene una visión tradicional de control (sin planificación) por la autoridad de tránsito local.

En el caso del peatón, la normativa provincial lo aborda en relación con el uso de la vía pública por motivos de seguridad vial y orden del tránsito; pero no lo reconoce como sujeto con derecho a la movilidad.

Por último, no se observa en la normativa analizada un tratamiento claro y coherente de los roles de cada uno de los agentes involucrados en la gestión del conjunto de modos que se utilizan en AMM.

\section{Regulación de los usos del espacio vial por modos motorizados y no motorizados}

Se observa que existe poca institucionalización de medidas tendientes a regular el uso del espacio vial por el vehículo particular o que incentiven el uso de modos no motorizados y motorizados colectivos. Además, no se identifica un tratamiento integral para el uso del espacio vial.

Así, para el estacionamiento medido, de un total de seis municipios que conforman el Área Metropolitana de Mendoza, solo tres cuentan con ordenanzas al respecto. Igualmente, ninguna normativa hace referencia a la disuasión del uso del auto particular e institucionalización de un tratamiento diferencial a favor de las personas. Es decir, no se cuenta con las bases para implementar sistemas de estacionamiento medido municipales que acompañen la gestión de la movilidad urbana sostenible.

Con respecto a la priorización de modos de transporte con bajo impacto ambiental, hay ordenanzas en los municipios de Maipú, Go- 
doy Cruz y Lujan de Cuyo. Maipú establece carriles exclusivos de transporte público de pasajeros en ciertas calles. Godoy Cruz declara de interés municipal el Proyecto Ferrotranvía Urbano, que proponía a nivel metropolitano la puesta en marcha de ferrotranvías urbanos ecológicos; y Luján de Cuyo declara de interés departamental las bicisendas existentes. Con respecto a la restricción a la circulación vehicular en ciertas áreas u horarios, solo dos municipios cuentan con normativa: Las Heras y Ciudad de Mendoza. La normativa de ambos municipios tiene como objeto regular el espacio vial para ordenar el tránsito vehicular y no así para tender hacia una restricción del uso del auto como medida de movilidad urbana sostenible.

Así, podemos afirmar que la regulación del uso de la vía pública municipal existente es escasa e insuficiente para lograr un desarrollo urbano orientado al transporte (DOT). Esto lo indica la no priorización de los modos de transporte menos contaminantes como "usuarios jerarquizados" en el uso de la vía pública.

\section{Regulación del tipo de información} disponible para los usuarios de los servicios de: transporte público colectivo, movilidad no motorizada y estacionamiento medido en el área metropolitana de Mendoza

Del conjunto de regulaciones para los diferentes medios de transporte considerados en el estudio, se identificó que el derecho a la información está garantizado para los usuarios del transporte público colectivo. El pliego licitatorio del año 2005 obliga a los concesionarios a brindar información en relación con los itinerarios, horarios, frecuencias y tarifas; y establece a través de qué medios debe implementarse, aunque no indica el modo en que el Estado provincial puede controlar que realmente sea cumplido.

Sin embargo, este derecho no está contemplado para los usuarios de los servicios de bicicletas, recientemente implementados tanto por el gobierno provincial como por los municipios de Ciudad de Mendoza y Godoy Cruz. Otro vacío normativo se encuentra para el usuario del sistema de estacionamiento medido de los municipios del AMM, donde no está contemplado por las ordenanzas que fijan la tarifa y el régimen de horarios. Ambos sistemas ameritan ser contenidos en una normativa provincial que permita armonizar los criterios para su regulación por los municipios.

Es fundamental comprender que la regulación e institucionalización de la gestión de la información al usuario de los servicios de transporte público colectivo, movilidad no motorizada y estacionamiento medido es clave en la implementación de un modelo de gestión de la movilidad urbana sostenible. Esto permite generar un sistema accesible y equitativo para la satisfacción de las necesidades de la población, en armonía con el ambiente, y eficiente desde el punto de vista económico.

\section{Síntesis dimensión político-institucional sobre la regulación de la movilidad urbana sostenible del área metropolitana de Mendoza}

De los indicadores observados para la dimensión político-institucional podemos afirmar que se evidencian grandes limitaciones 
para alcanzar criterios de movilidad urbana sostenible.

En términos de articulación intergubernamental para la planificación de la movilidad urbana sostenible, se identifica un pobre diseño institucional que la facilite. Esto se agrava si se considera la complejidad de la articulación interjurisdiccional propia de los Estados federales. Esto último puede ser la variable explicativa al pobre diseño institucional señalado.

En relación con la regulación del uso de los diferentes modos de transporte, se observa que la legislación municipal vigente para el área metropolitana de Mendoza no responde a criterios de movilidad urbana sostenible y, a su vez, presenta diferencias notables entre sí. Además, el conjunto de ellos responde al esquema tradicional de gestión del transporte que se organiza con un enfoque "modal" y no al esquema integral que propone el paradigma de la movilidad urbana sostenible que además pone a la persona como sujeto de derecho.

Sobre la regulación del espacio vial por modos motorizados y no motorizados, si bien la planificación, la gestión y el mantenimiento de la vialidad urbana son competencia municipal, se observa una ausencia en la normativa provincial que permita a la autoridad coordinadora armonizar los criterios de su gestión. Los municipios de Ciudad de Mendoza, Godoy Cruz y Maipú presentan mayores avances al respecto, aunque responden a las nociones tradicionales de control del tránsito, restricciones y usos exclusivos focalizados, y no a la visión integral de la movilidad urbana sostenible.
Por su parte, la regulación del tipo de información disponible para los usuarios de los servicios de transporte público colectivo, movilidad no motorizada y estacionamiento medido es muy limitada. Si bien es reconocido el derecho al acceso a la información por el usuario de transporte público colectivo, el mismo no está contemplado para los usuarios de los servicios de bicicletas y de estacionamiento medido.

\section{Indicadores de la relación entre el sistema de movilidad y el sistema urbano}

\section{Tributación diferenciada para el suelo urbano vacante (suv)}

El actual modelo de expansión urbana del AMм se desarrolla hacia la periferia, dejando suelo vacante entre las construcciones que poseen servicios públicos y los nuevos desarrollos inmobiliarios. Para alcanzar las nuevas construcciones, los servicios deben extenderse a través de las áreas vacantes que se benefician con los servicios y equipamientos. En este sentido, interesa conocer las características del tributo inmobiliario sobre el suelo urbano vacante, con el fin de determinar si la regulación de terrenos baldíos favorece la prestación de servicios públicos.

En lo que respecta al impuesto inmobiliario, el Código Fiscal Provincial establece uno adicional a los terrenos baldíos (aquellos que no están edificados o cuyas construcciones no son habitables) ubicados en zonas urbanas indicando que, además del impuesto correspondiente, se pagará conjuntamente con el 
tributo un monto fijado por la Ley Impositiva. Es decir, que el tipo de tarifa para el suv es diferencial.

La Ley Impositiva y de Avalúo 8837/2016 establece el cálculo de los importes fijos, alícuotas, tasas y contribuciones correspondientes al impuesto inmobiliario. En su artículo 2 fija la fórmula de aplicación de alícuotas en función del valor del inmueble (a mayor valor mayor alícuota) y su localización (mayor valor para los ubicados en zonas urbanas). El adicional al baldío se determinará aplicando una fórmula que relaciona un adicional mínimo y máximo (250 y $500 \%$ ) con los avalúos mínimos y máximos fijados anualmente.

Por otro lado, se fija que la base de cálculo de avalúo fiscal será la resultante del valor del terreno y las mejoras. Las mejoras edilicias se determinan según puntaje de categorización de materiales de construcción, antigüedad, valor residual y superficie del polígono construido. Sin embargo, si bien los terrenos baldíos sufragan un adicional, no pagan el porcentaje de mejoras adquiriendo de igual manera los beneficios de las obras públicas y privadas de su entorno. Este último punto constituye una limitación, ya que a pesar del pago del adicional, mantener suelo vacante continúa siendo favorable desde el punto de vista de la especulación inmobiliaria dado el aumento de valor que adquiere la propiedad a partir de las mejoras de su entorno.

La Ley 8051 de Ordenamiento Territorial y Usos de Suelo propone una coordinación interministerial en materia fiscal. En su artículo 54 expone acciones dirigidas a desalentar la especulación inmobiliaria y promover la inversión por medio de la adecuación de los instrumentos fiscales.

Se observa que la provincia de Mendoza presenta una tributación diferencial para los terrenos baldíos, si bien esta situación se presenta como favorable, no resulta efectiva en términos del desarrollo equitativo y sostenible de la ciudad en relación con la eficiencia de prestación de servicios. En este sentido, la aplicación de sobretasas podría funcionar como un instrumento que busca desalentar su existencia e incentivar las inversiones; sin embargo, es fundamental considerar que el fenómeno es resultado de múltiples causas, por lo que se requiere de un análisis más profundo para inducir conductas sobre el desarrollo urbano a través de la tributación.

\section{Intensidad de ocupación y zonificación de usos}

La dinámica de ocupación para el AMM se presenta compacta en el centro y dispersa hacia la periferia, configurando un modelo de expansión urbana de baja densidad. Comprender la configuración territorial a partir de la relación entre índices de ocupación, superficies de parcela, densidad de población y zonificación de usos de suelo nos permite vislumbrar si el modelo territorial contribuye o no con el paradigma de movilidad urbana sostenible.

Como se mencionó, el AMM está constituida por seis municipios y ordenanzas de zonificación con criterios que varían de un municipio a otro, resultando en una ocupación desordenada del territorio. Las áreas 
periurbanas siguen siendo consideradas zonas agrícolas, sin vislumbrar los cambios de usos que genera la expansión urbana sobre las mismas (necesidad de actualización de usos) y las superficies mínimas permitidas para los nuevos loteos, en algunos casos, varían entre los 500 y 1000 metros cuadrados. Se visualiza la necesidad de normativa que se adecue a la dinámica territorial actual ya que no todos los municipios especifican usos permitidos por zonas como tampoco valores de FOS y FOT por zona y por actividad. Por otro lado, se presentan sectores sin uso definido, lo que incentiva el asentamiento de actividades desprovistas de las condiciones mínimas necesarias para su desarrollo.

La baja densidad de población complementada con falta de definición de usos y actividades parece ser un indicador incompatible con la movilidad urbana sostenible.

\section{Distribución de usos del suelo}

La localización de actividades dentro de la trama urbana condiciona la selección de los modos de transporte. La expansión hacia la periferia en el Амм propicia el uso del automóvil particular. Las grandes distancias al centro de la ciudad donde se concentran las funciones administrativas, de comercio y servicios obligan a la población a trasladarse para satisfacer la demanda.

La periferia se constituye en un uso predominantemente residencial de barrios de tipo privado y cerrado, que ocupan gran parte de tierras de cultivo, en forma de ramificación, siguiendo los accesos principales y conformando islas fragmentadas de la mancha consolidada. Se observa que las cabeceras de los departamentos de Maipú, Luján de Cuyo y Guaymallén han comenzado a desarrollarse

FIGURA 2. USOS DEL SUELO PARA EL ÁREA METROPOLITANA DE MENDOZA

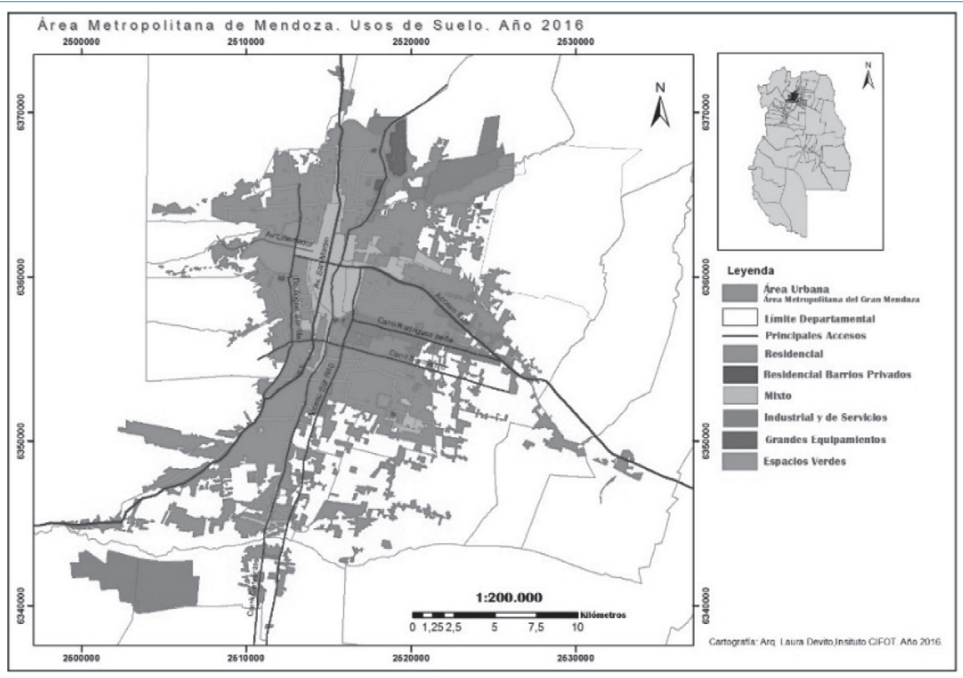

Fuente: cartografía, Arq. Laura Devito, Instituto CIFOT, 2016. 
como centros de uso mixto. Al norte, centro y sur se localizan sectores industriales concentrados, en muchos casos conviviendo con actividades de uso incompatible.

La Ley 8051 establece como objetivo específico la planificación de usos de suelo compatibles para evitar conflictos territoriales, impidiendo la pérdida de espacio público y la fragmentación del territorio. Define pautas para implementar un modelo de gestión sistémico del territorio a partir de una visión integral y estratégica a escala provincial y municipal. La visión del territorio como sistema contribuye a promover la articulación entre la planificación urbana y el sistema de transporte público, y a valorar la mezcla de funciones y usos urbanos en un mismo espacio buscando generar patrones de proximidad para evitar desplazamientos motorizados. Este último punto se acerca a la concepción de la movilidad urbana sostenible.

\section{Síntesis de los indicadores que dan cuenta de la relación entre el sistema de movilidad y el sistema urbano}

Los indicadores analizados demuestran que el Estado cuenta con instrumentos normativos de la planificación urbana que inciden en la regulación del modelo de movilidad. Sin embargo, presentan limitaciones en su aplicación y control lo que dificulta la implementación del paradigma de movilidad urbana sostenible.

El AMm presenta un modelo de expansión hacia la periferia a partir de un centro altamente consolidado, configurando un modelo de expansión urbana de baja densidad que presenta grandes zonas intersticiales de suelo vacante. En el caso del servicio de transporte colectivo, la existencia de terrenos baldíos incide en la extensión de redes y recorridos, incremento en costos operativos y aumento en el tiempo de espera y traslado. Es por esto que la planificación urbana debe considerar el tratamiento del suelo vacante mediante instrumentos fiscales regulatorios como las sobretasas diferenciales del tributo, la bonificación por zonificación o bonos de densidad, entre otros. Al decir de Pizarro (2013), "La estructura de la fiscalidad de suelos e inmobiliaria tiene un efecto directo en la distribución espacial de las actividades comerciales y productivas. Siendo esto lo que más incide en la demanda de movilidad urbana en el mediano y largo plazo".

Si bien en la provincia de Mendoza existe una vasta legislación referida a los temas ambientales (Ley de Aguas y Ley de Medio Ambiente), hasta la sanción de la Ley de Ordenamiento Territorial y Usos del Suelo (Ley 8051/09), solo existían reglamentaciones parciales que han contribuido a que el avance urbano se hiciera en forma descontrolada aumentando el deterioro ambiental y generando externalidades negativas (Gudiño, 2010).

La Ley 8051 establece que cada municipio elaborará su plan de ordenamiento territorial donde quedarán establecidas las zonificaciones y los factores de ocupación del territorio bajo un modelo de ocupación común, sin admitir usos y tipos de edificaciones incompatibles, es por esto que la concepción sistémica del territorio beneficiaría la prestación de servicios de mayor calidad. 
Se observa la existencia de instrumentos de regulación como el tributo diferencial, los factores de ocupación del suelo, las zonificaciones municipales y la Ley 8051, lo que da cuenta de la existencia de un marco normativo sobre las acciones que se realizan en el territorio. Sin embargo, los instrumentos antes citados, salvo la Ley 8051, están planteados de manera rígida y sectorial. Su limitación se encuentra en que comprenden el territorio de manera sectorial y no existen mecanismos de control que efectivicen su aplicación. Tampoco se observa la presencia de objetivos dirigidos a promover la movilidad urbana sostenible.

Por esto, se debe prever la planificación territorial del sistema de transporte enmarcado en el paradigma de movilidad urbana sostenible, orientada a facilitar el acceso de la población a los servicios y equipamientos básicos. Para ello, la planificación urbana y del modelo de movilidad debe articularse a partir de objetivos comunes basados en un marco normativo integral que regule la organización del territorio. Esto será posible a partir de una gestión que coordine y controle las acciones de los distintos agentes que participan en las decisiones y acciones sobre el territorio provincial.

\section{Indicadores del esquema de financiamiento}

\section{Integración tarifaria entre modos}

Un sistema tarifario integrado debe cumplir las siguientes condiciones: a) coordinación de los recorridos de los modos de transporte, b) tarifa única para realizar el desplazamien- to y c) trasbordos sin costo (limitados o ilimitados en: cantidad, franja horaria y área determinada).

En el servicio de transporte del Амм la integración tarifaria se da de hecho ya que no figura en la legislación vigente. La Ley 7412, que hace referencia al régimen tarifario, determina que debe ser única y exclusivamente fijada por la autoridad competente del Poder Ejecutivo, pero no se alude a la integración tarifaria. Esto está justificado porque la mencionada ley es una legislación marco y por ello contiene conceptos generales.

La integración tarifaria existente se da entre ómnibus, trolebús y tranvía; y la misma no se sigue de manera estricta ya que no se cumple la primera de las condiciones. La coordinación de los recorridos de los distintos modos de transporte no se considera de ninguna manera en la planificación del servicio. No obstante, se considera que existe integración tarifaria aunque esta no logra generar todos los beneficios que la caracterizan al cumplirse la primera condición. Se alcanza, entonces, la situación deseada para la movilidad urbana sostenible de presencia de integración tarifaria.

Adicional a este análisis debe considerarse que la integración tarifaria merece un estudio pormenorizado de los costos de los distintos modos, sin dejar de lado el automóvil particular y las externalidades de todos ellos, como también un estudio de la demanda de transporte y movilidad. En el presente caso, solamente se cuenta con estudios parciales de los modos colectivos e individuales (a través de las encuestas de origen y destino de los años 1991, 1998, 2005 y 2010) pero nada existe aún sobre movilidad. 


\section{Trasbordos sin costo}

El servicio de transporte colectivo del AMM tiene un sistema de trasbordos que permite combinar dos modos o dos recorridos en el mismo modo (ómnibus, trolebús o tranvía) para llegar al destino, abonando un solo pasaje. El sistema opera de la siguiente manera: una vez abonada la tarifa se puede realizar un trasbordo en un periodo no mayor a 90 minutos.

Este indicador da cuenta de la presencia de trasbordos, es decir, se observa una situación favorable. Sin embargo, para poder afirmar esto de forma contundente es necesario conocer la cantidad de trasbordos que se realizan en todo el sistema y detectar si son los adecuados en función del tiempo de viaje y de la cobertura del servicio. Este análisis no se ha realizado para el servicio de transporte del AMM, por lo que no se conoce el impacto de los trasbordos implementados.

Asimismo, se observa que el mecanismo de trasbordos del servicio de transporte del área en estudio permite no solamente el cambio de un modo de transporte a otro o de un recorrido a otro en el mismo modo de transporte, es decir, completar un desplazamiento, sino también la utilización del trasbordo para el viaje de regreso. De esta manera, al no existir información respecto del nivel de trasbordos no se puede conocer qué cantidad de los mismos corresponde al regreso a casa y, por tanto, no cumplen con el propósito de los mecanismos de trasbordos que es completar un viaje o desplazamiento.

\section{Recaudación / distribución por único operador}

El servicio de transporte colectivo mediante ómnibus realiza su recaudación a través de un único operador. Diariamente, dicha recaudación se distribuye entre los operadores de transporte según los kilómetros recorridos, previamente se descuentan las tasas e impuestos. De esta manera, se afirma la presencia de un único recaudador/operador.

Esta es una característica favorable del servicio ya que deja a las empresas concesionarias sin riesgo en la operación del servicio. Es decir, deben remitirse a realizar sus actividades pautadas con la autoridad de aplicación sin preocuparse por los pasajeros transportados (no asumen riesgo de demanda).

La existencia de un único recaudador/distribuidor no consta en la normativa vigente, sino que es una medida tomada por la autoridad de aplicación a partir de la implementación del sistema de cobro con tarjeta prepaga. El cobro previo a la realización del servicio permite la recaudación anticipada y la distribución de la misma entre los operadores del servicio.

\section{Proporción de financiamiento mediante subsidios (proporción de financiamiento mediante tarifa)}

En el año 2012, el gasto total en el servicio de transporte colectivo del Амм fue de $\$ 747.711 .000$, este gasto fue afrontado en más de un $60 \%$ por subsidios nacionales y provinciales, y el $40 \%$ restante por la recaudación del sistema. Es decir, el indica- 
dor proporción de financiamiento mediante subsidios es de 0,6 y el indicador proporción de financiamiento mediante tarifa es de 0,4 . Estos valores dan cuenta de una situación satisfactoria.

Es necesario aclarar que este indicador debe ser complementado con indicadores relativos a la calidad del servicio para así asegurar que los subsidios alcancen el objetivo de proveer un servicio que beneficia a toda la sociedad. En el caso del servicio de transporte del área en estudio podría pensarse que se requieren aún medidas que mejoren la calidad del mismo.

A su vez, es de gran importancia considerar este modo de transporte en relación con otros modos y analizando no solamente los subsidios directos. Por ejemplo, al evaluar el automóvil particular y la gran cantidad de externalidades negativas que genera (congestión vehicular, contaminación, uso irracional de recursos escasos) podría afirmarse que la sociedad en su conjunto soporta o "subsidia» a los usuarios de dicho modo ya que ellos no "pagan" por estos males que le producen a la sociedad. Sin embargo, estos temas exceden la finalidad del presente trabajo.

\section{Organismo que fija la tarifa}

En el servicio de transporte colectivo del AMM el organismo que fija la tarifa y la regula es de carácter público según figura en la Ley 7412 del ańo 2005, en los pliegos licitatorios del año 2005 y en la Ley 6082 del año 1993.

De esta manera, se alcanza la situación deseada de que la tarifa la defina un ente público que vele por las necesidades de todos los actores involucrados y no los operadores privados que siguen criterios de rentabilidad y de mercado competitivo.

Este indicador está vinculado al indicador de proporción de financiamiento mediante subsidios ya que en una situación de incremento de costos (operativos y de infraestructura) si la tarifa se deja fija, limita la recaudación y requiere de mayor nivel de subsidios.

\section{Síntesis de los indicadores de la dimensión del financiamiento y la tarificación}

En la dimensión económica se detecta que el Estado provincial cuenta con los instrumentos mínimos necesarios para promover la movilidad urbana sostenible. Los indicadores analizados dan cuenta de una situación favorable, con grandes potencialidades y algunas limitaciones respecto de las posibilidades de acción del Estado.

La integración tarifaria opera como un adecuado instrumento de regulación. Permite favorecer a quienes requieren de trasbordos para completar su desplazamiento que, en general, son personas que necesitan realizar recorridos de mayor distancia. Estas personas suelen pertenecer al grupo poblacional que no posee un modo de movilidad motorizado particular (moto o automóvil) y que residen en territorios más alejados de los centros de mayor actividad. La gran limitación que tiene la integración tarifaria es la falta de integración y jerarquización de los modos de transporte y sus recorridos. Por su parte, la gran potencialidad es efectivizar la integración de modos e incluir a aquellos excluidos como son la bicicleta y los motorizados particulares. 
Los trasbordos sin costo operan también como un instrumento favorable a la movilidad urbana sostenible. Nuevamente, la limitación resulta ser la falta de jerarquización de recorridos y la integración de modos que facilitarían el desplazamiento en tanto podrían reducir tiempos de viaje y favorecer a los usuarios. Se podría así hacer mejor uso de las ventajas de los trasbordos sin costo. La potencialidad de este aspecto, al igual que en el indicador anterior, es el beneficio que se podría generar a quienes residen más lejos de los centros de actividad ya que podrían no solo favorecerse por el menor pago de tarifa sino también por el menor tiempo de viaje producto de la coordinación entre modos y recorridos.

La recaudación / distribución por un único operador resulta también beneficiosa en términos de promover el enfoque de la movilidad urbana sostenible. De esta manera, el Estado tiene pleno control de los fondos que ingresan por concepto de tarifa y puede calcular así los requerimientos de financiación del servicio. La limitación detectada es que, en este contexto, el Estado asume la totalidad del riesgo de demanda. Este hecho se reconoce como favorable a la movilidad urbana sostenible ya que es un servicio público y, como tal, el Estado debe garantizar su adecuado funcionamiento. Las empresas, entonces, no deben preocuparse por la demanda del servicio ya que los costos que no se soportan con la tarifa los asume el Estado. Sin embargo, tal como está planteado en el servicio de transporte colectivo del AMM, las empresas operadoras no tienen ningún incentivo para prestar un servicio de calidad. Es decir, esta medida debería acompañarse por controles o exigencias que aseguraran una mejor operación del servicio. Si bien existen controles respecto de la antigüedad del parque móvil y del mantenimiento de los vehículos, nada se exige respecto del uso de tecnologías con bajas emisiones contaminantes o respecto de la comunicación y señalización adecuada del servicio. La gran potencialidad de que la distribución se realice por un único operador es que el Estado puede definir las condiciones de distribución. En la actualidad, la misma se realiza por kilómetro recorrido, pudiendo determinarse que se haga por una combinación entre kilómetros recorridos y pasajeros transportados. De esta manera, podrían establecerse incentivos a las firmas concesionarias para mejorar la calidad del servicio.

La proporción de financiamiento mediante subsidios es del $60 \%$ y en el contexto de la movilidad urbana sostenible esto es satisfactorio ya que implica que más de la mitad de los costos del servicio son soportados por toda la sociedad que se beneficia del mismo. Sin embargo, estos subsidios están compuestos en un $40 \%$ por subsidios provinciales y en un $60 \%$ por subsidios nacionales, quedando bajo discusión, entonces, que la mayor parte de la carga le corresponde a la nación y no todos los habitantes de la misma se benefician por el servicio de transporte del AMM. Este hecho da cuenta, a su vez, de una fuerte dependencia de los fondos nacionales, lo que resulta una limitación de las capacidades institucionales provinciales para regular el sistema de transporte colectivo. Otra de las limitaciones, es la falta de calidad del servicio en lo operativo. Si la dimensión operativa no es óptima, si el servicio no presenta aspectos operativos efi- 
cientes y pensados para el usuario, la sociedad en su conjunto estaría financiando un servicio de baja calidad que no resulta en un beneficio para todos. La potencialidad de este instrumento se detecta en las posibilidades de demandar a la empresas prestadoras del servicio una mejora en la calidad del mismo, el Estado puede a través de este instrumento definir incentivos para realizar acciones superadoras de la calidad. Asimismo, el nivel provincial puede coordinarse con el nivel nacional en la definición de dicho los incentivos.

Por último, el organismo que fija la tarifa es el estado provincial, dotándolo de la posibilidad de definir cuánto se financia mediante tarifa y cuánto mediante subsidios. Es decir, es un instrumento que favorece al enfoque de la movilidad urbana sostenible. Este indicador está vinculado al de proporción de financiamiento mediante subsidios ya que en una situación de incremento de costos (operativos y de infraestructura), si la tarifa se deja fija, limita la recaudación y requiere de mayor nivel de subsidios. La limitación de este instrumento es el alto costo político e institucional de modificar la tarifa, dado que un aumento de la misma podría implicar un alto costo en términos de la aceptación de la medida por parte de los usuarios. A su vez, si dicho aumento solo implica disminución de los subsidios provinciales se generaría un costo institucional de coordinación con el nivel nacional. La gran potencialidad de este instrumento es la posibilidad de decidir el nivel tarifario siempre en beneficio de los usuarios, privilegiando el transporte colectivo y desincentivando el uso del automóvil particular.
En síntesis, si bien la dimensión económica cuenta con los instrumentos mínimos necesarios para propender por una movilidad urbana sostenible, es necesario que esta se vincule y coordine con otras dimensiones tales como la operacional del servicio y la institucional que garanticen que los instrumentos se utilicen adecuadamente.

\section{CONCLUSIONES GENERALES $Y$ LINEAMIENTOS DE POLÍTICA PÚBLICA}

El sistema de movilidad urbana del AMM se encuentra regulado por la jurisdicción provincial y municipal. Dicha regulación está provista de grandes aciertos pero también de muchas limitaciones. La mencionada regulación se expresa en instrumentos, por lo cual se evaluó si el Estado provincial y los municipios que conforman el área de estudio cuentan con ellos.

En una primera aproximación podemos afirmar que hay instrumentos, pero que presentan limitaciones y falencias en su perspectiva y tratamiento de los usos de los modos de transporte, los usos del suelo y el financiamiento.

En la dimensión político-institucional es preciso definir el rol de los actores principales que intervienen en la implementación de la movilidad urbana sostenible, para ello es primordial abordar la regulación a partir de un consenso entre el Estado provincial, los municipios, el sector privado involucrado en la prestación del servicio de transporte y los usuarios, a fin de coordinar acciones ten- 
dientes al desarrollo de la movilidad urbana sostenible.

Se sugiere que el Estado provincial defina las competencias en materia de regulación de los modos de transporte a nivel provincial y municipal estableciendo lineamientos dirigidos a una planificación integral del transporte existente tendientes a la movilidad urbana sostenible. Por otro lado, se propone que los modos de transporte sostenibles, ciclovías y tranvía, sean tratados como servicios públicos; de esta manera, el Estado deberá asegurar a los ciudadanos la satisfacción de la necesidad de movilidad y traslado por medio del uso de modos limpios, es decir, que hay un compromiso político implicado en la prestación del servicio.

En lo que a los usuarios se refiere, es importante focalizar que el rol de estos tiene relación con la elección de modos de transporte para realizar sus actividades diarias y el impacto negativo o positivo que esta elección supone. Es primordial, entonces, contar con adecuada información y un eficiente sistema de movilidad que articule distintos modos de transporte. En términos de regulación, este derecho está garantizado solo para los usuarios del transporte público de pasajeros sin abarcar otras modalidades. A la hora de promover un sistema de movilidad urbana sostenible se hace necesario contar con instrumentos de control que permitan asegurar que este derecho se cumpla y con instrumentos normativos que incorporen el derecho a usuarios de otros modos de transporte con el objeto de promover la multimodalidad. Es notable apreciar que el abordaje en materia de regulación no es igual para todos los modos ni en todas las jurisdicciones, ya que la integralidad de modos no está presente; así, el transporte público de pasajeros presenta una buena regulación pero esto no se observa para el servicio de bicicletas (tanto para su alquiler como su estacionamiento), motos o estacionamiento medido para automóviles particulares. La implementación de un sistema de movilidad urbana sostenible supone proponer, además de instrumentos de regulación, un sistema de incentivos dirigido a aumentar los viajes en transporte público y no motorizado a fin de reducir las externalidades negativas, del mismo modo que haga visible la responsabilidad de los distintos actores sociales y el impacto de sus decisiones.

En lo que respecta a la dimensión urbana del sistema de transporte, en la regulación de usos de suelo las limitaciones se encuentran en una ausencia de criterios comunes de ordenación del territorio a nivel municipal y provincial, lo que resulta en una conformación territorial que incentiva el uso del automóvil particular a partir de una distribución de actividades nucleadas principalmente en el centro de la ciudad, acompańado de un crecimiento de baja densidad hacia la periferia. La necesidad resulta en contar con instrumentos municipales como las zonificaciones de usos de suelo que sean acordes a la configuración territorial actual y respondan a una visión integral del territorio considerando los modos de transporte y la distribución de actividades como estructurantes de la dinámica de desplazamientos. Es decir, abordar la planificación y la movilidad urbana de manera integral, alineando las políticas y estrategias a partir de la coordinación de los distintos organismos que 
se encargan de llevarlas a cabo y garantizando su continuidad en el largo plazo.

Por su parte, la dimensión económica es la que cuenta con los instrumentos mínimos necesarios que le permiten a la autoridad reguladora ejercer esta función. Para su análisis se desarrollaron cinco indicadores. La integración tarifaria se da entre los modos colectivos y resulta muy favorable para quienes requieren desplazarse distancias más largas, al igual que los trasbordos sin costos. La recaudación / distribución por un único operador favorece el desarrollo de la movilidad urbana sostenible, dando al Estado el control total de los fondos que ingresan por concepto de tarifas, conociendo perfectamente, entonces, los requerimientos de financiación del servicio. Sin embargo, este factor carga al Estado con todo el riesgo de demanda, por lo que no se generan los incentivos para que las empresas operadoras busquen atraer a nuevos usuarios. Asimismo, se observa que el financiamiento mediante subsidios alcanza un valor favorable en términos de la movilidad urbana sostenible, aunque gran parte de ese fondo proviene de la nación. Es decir, la provincia, que es quien regula al sistema de transporte, pierde capacidad de decisión frente a este escenario. Finalmente, se alcanza la situación deseada de que la tarifa la defina un ente público que vele por las necesidades de todos los actores involucrados y no los operadores privados que siguen criterios de rentabilidad y de mercado competitivo.

Estos indicadores podrían implicar mejor desempeño del sistema de transporte si contaran con una coordinación y jerarquización de los recorridos del transporte colectivo y si se incorporaran a la red los modos no motorizados como la bicicleta y la caminata. De esta manera se podrían reducir los tiempos de viajes y mejorar la calidad de la movilidad para quienes viven en las áreas periféricas. Los residentes de estas áreas son grupos homogéneos, o de alto poder adquisitivo o de bajos recursos, los primeros basando su movilidad en el automóvil particular, los segundos dependiendo casi exclusivamente de los modos públicos. Por ello, la mejora en la movilidad mediante modos colectivos y no motorizados resulta un gran beneficio para los grupos poblacionales de menores ingresos. La regulación en la dimensión económica requiere, entonces, de una adecuada regulación en las otras dimensiones, sobre todo de la planificación de la red de transporte y del sistema urbano.

\section{REFERENCIAS}

Acevedo, J. y Bocarejo, J. P. (2009). Movilidad sostenible: una construcción multidisciplinaria. Revista de Ingeniería, Universidad de Los Andes, 29, 72-74.

Agencia de Ecología Urbana de Barcelona (2010). Plan de Indicadores de Sostenibilidad Urbana de Vitoria-Gasteiz. Barcelona: BCN Ecología.

Asociación Unida Transporte Automotor Mendoza (2009). Costos y tarifas del transporte urbano de pasajeros. Mendoza, Argentina.

Cohen Arazi, M. (2012). La tributación diferenciada del suelo urbano como herramienta para el desarrollo: aplicación al caso de Córdoba, Argentina. $45^{\circ}$ Jornadas Internacionales de Finanzas Públicas, Universidad Nacional de Córdoba, Argentina. 
Comisión Europea (2007). Libro Verde del Transporte Urbano Hacia una nueva cultura de la movilidad urbana. Ginebra: com.

Figueroa, O. y Orellana, A. (2007). Transantiago: gobernabilidad e institucionalidad. Revista latinoamericana de estudios urbano-regionales, EURE, XXXIII (100).

Forray Claps, R. (2013). Transantiago: ¿Qué perseguia la decisión pública? La fábrica del movimiento. 16 casos de politicas públicas para la movilidad urbana. Ciudad Autónoma de Buenos Aires: Café de las ciudades.

Fredani, J. (2014). Tierra vacante y desarrollo urbano en ciudades medias. Estrategias de recuperación e integración territorial en el gran La Plata, Argentina. VI Seminario Internacional de Investigación en Urbanismo, Barcelona.

García Schilardi, M. E. (2014). Transporte Público Colectivo: su rol en los procesos de inclusión social. Revista Bitácora (24), 35-42.

García Schilardi, M. E. (2013). Organización territorial urbana y transporte público masivo: metrópolis del Gran Mendoza. Urbano (28), 50-57.

Gudiño, M. E. (2010). Del urbanismo reglamentario a las nuevas concepciones de ordenamiento territorial. Actas del xi Coloquio Internacional de Geocrítica: La planificación territorial y el urbanismo desde el diálogo y la participación, Buenos Aires.

Holigue, C. (2011). Institucionalidad y transporte público urbano: Santiago de Chile y Medellín, Colombia. Innovación ambiental de servicios urbanos $y$ de infraestructura: hacia una economia baja en carbono. Santiago de Chile: CePAL-un. Agencia Española de Cooperación Internacional para el Desarrollo (AECID).

Lindblom, Ch. (1991). El proceso de elaboración de politicas públicas. Madrid: INAP.
Losada, F. (2013). Algunas notas en torno a determinados servicios de transporte terrestre de pasajeros en la provincia de Mendoza. Mendoza (inédito).

Losada, F. (2015). Conceptos para la regulación del transporte y la movilidad. Ciclo de formación en gestión de la Movilidad Urbana Sostenible. Mendoza (inédito).

Martínez, L. (2015). Las rupturas y continuidades en el marco regulatorio del servicio de transporte público de pasajeros y el rol del Estado en Mendoza en el periodo 1991-2013 [Tesis de Licenciatura en Ciencia Política y Administración Pública]. Mendoza: Facultad de Ciencias Políticas y Sociales.

Ministerio de Transporte de la Provincia de Mendoza (2010). Plan integral para la movilidad para el Gran Mendoza 2030. Mendoza: Ministerio de Transporte.

Ministerios de Transporte (2015). Propuesta para la movilidad y el transporte. Ejes de análisis provincial de Mendoza. Mendoza: Ministerio de Tranporte.

Muñoz, M., Juan, P. y Anguita Rodríguez, F. (2013). Marco normativo de movilidad sostenible en España. España.

Pando, D. (2006). Capacidad de gestión estatal en la regulación de servicios públicos privatizados: telecomunicaciones, agua, gas natural y electricidad en Argentina (1990-2001) [Tesis doctoral en Gobierno y Administración Pública]. Madrid: Instituto Universitario de Investigación Ortega y Gasset, Universidad Complutense de Madrid.

Pereyra, A. (2006). Alternativas en el manejo del riesgo de demanda en concesiones de infraestructura vial. Uruguay: Departamento de Economía. Facultad de Ciencias Sociales. Universidad de la República de Uruguay. 
Pizarro, A. (2013). Políticas integradas y sostenibles de movilidad: revisión y propuesta de un marco conceptual. Boletin FAL, CEPAL, 323 (7).

Roa, H. (2013). Movilidad urbana e indicadores de exclusión social del sistema de transporte: evidencia de una ciudad intermedia chilena. Revista Transporte y Territorio, (8), 45-64.

UITP (2004). Ticket to the Future: Three Stops to the Sustainable Mobility. International Association of Public Transport..

Vicentini, V. (2013). Los nuevos paradigmas de la movilidad urbana sostenible. Banco Interamericano de Desarrollo. Presentación en I Congreso de Transporte, Sustentabilidad y Ordenamiento Territorial, Mendoza, Argentina.

Zaragoza, A., Thorson, O., Serrano, A., Rodríguez, J., Zamorano, C. y Aurin, R. (2009). Diálogo sobre movilidad urbana. Ingeniería y Territorio (86), 92-105.

Jurisprudencia

Código Fiscal de Mendoza (2015). Administración Tributaria Mendoza.

Código Fiscal de Mendoza (2016). Administración Tributaria Mendoza.

España. Jefatura del Estado. Ley Nacional 2/2011 de Economía Sostenible. BoE, núm. 55, de 5 de marzo de 2011. Referencia: воE-A-2011-4117.

Gobierno de Mendoza. Ministerio de Ambiente y Obras Públicas (2010). Encuesta de Origen y Destino 2010. Mendoza, Argentina.
Provincia de Mendoza. Gobierno de Mendoza. Ley de Loteo 4341/1979.

Provincia de Mendoza. Gobierno de Mendoza. Ley de Tránsito y Transporte 6082/1993.

Provincia de Mendoza. Gobierno de Mendoza. Ley Marco sobre la Administración, Planificación y Regulación del Transporte Público de Pasajeros, en todas sus formas y modalidades, 7412/2005.

Provincia de Mendoza. Honorable Legislatura de la Provincia de Mendoza. Ley de Ordenamiento Territorial y Usos del Suelo. 8051/2009, artículos 7, 14,21 y 24.

Provincia de Mendoza. Gobierno de Mendoza. Ley de Administración Tributaria Mendoza, $8521 / 2013$.

Provincia de Mendoza. Gobierno de Mendoza. Ley Orgánica de Municipalidades, 1079

Provincia de Mendoza. Gobierno de Mendoza. Ley de Avalúo e Impositiva Mendoza, 8777/2014.

Provincia de Mendoza. Gobierno de Mendoza. Ley de Avalúo e Impositiva Mendoza, 8778/2015.

Provincia de Mendoza. Gobierno de Mendoza. Ley de Avalúo e Impositiva Mendoza, 8837/2016. Boletin Oficial de la Provincia de Mendoza. Mendoza. Argentina.

Provincia de Mendoza. Gobierno de Mendoza. Ley de Ministerios 8385/2011. Boletín Oficial de la Provincia de Mendoza. Mendoza. Argentina. 